\title{
DUPIRE'S EQUATION FOR BUBBLES
}

\author{
ERIK EKSTRÖM ${ }^{1}$ AND JOHAN TYSK ${ }^{1}$
}

\begin{abstract}
We study Dupire's equation for local volatility models with bubbles. The equation for call options contains extra terms compared to the usual equation, whereas, surprisingly enough, the Dupire equation for put options does not contain any extra terms. We also note that uniqueness of solutions to the Dupire equation is lost in general, and we show how to single out the option price among all possible solutions.
\end{abstract}

\section{INTRODUCTION}

Financial bubbles have been studied extensively over the last few years, see for instance [4], [6], [7], [9], [11] and [12]. It has been suggested to use models in which the underlying discounted price process is a strict local martingale under the pricing measure. Such models are known to exhibit several anomalies. For example, a call option price is not necessarily convex as a function of the spot price of the underlying, the put-call parity takes a different form, and the uniqueness of solutions to the corresponding BlackScholes equation is lost.

The Dupire equation is a forward equation for the call option price $C$ as a function of the strike price $K$ and the time to maturity $T$. It is argued in [5] that if the underlying stock price process follows a local volatility model, then the call option price satisfies

$$
\left\{\begin{array}{l}
C_{T}(K, T)=\mathcal{L} C(K, T) \quad \text { for }(K, T) \in(0, \infty)^{2} \\
C(K, 0)=(x-K)^{+}
\end{array}\right.
$$

where $\mathcal{L}$ is the second order differential operator

$$
\mathcal{L}=\frac{\sigma^{2}(K, T)}{2} \frac{\partial^{2}}{\partial K^{2}}-(r-q) K \frac{\partial}{\partial K}-q
$$

Here $r$ is the interest rate, $q$ is the continuous dividend yield, $x$ is the current stock price and $\sigma$ is the local volatility function. Since call prices for different strikes and maturities are observable quantities, the Dupire equation is commonly used to recover the volatility from $C$ and its derivatives with respect to $K$ and $T$. In the case of bubbles, however, it is easy to check that the Dupire equation fails in its usual form, see below. In the present paper we consider Dupire type equations for local volatility models with bubbles. In our main result, Theorem 2.2, we show that the Dupire equation for call

Date: October 13, 2009.

2000 Mathematics Subject Classification. Primary 91B28, 60G48; Secondary 35A02, $60 \mathrm{~J} 60$.

Key words and phrases. Dupire's equation; strict local martingales; financial bubbles.

JEL Classification. C02, G12.

${ }^{1}$ Support from the Swedish Research Council is gratefully acknowledged. 
options in this case contains extra terms. Surprisingly, the corresponding equation for put options does not contain these extra terms, and is therefore perhaps better suited for calibration issues. As is well-known for the corresponding Black-Scholes equation for bubbles, see [7], special care is needed to ensure the uniqueness of solutions. We show that the option price is the unique classical solution of the Dupire equation with a bounded distance to the pay-off function.

Even though the Dupire equation is of both theoretical interest and of practical use, the academic literature is somewhat sparse. In [13], the Dupire equation is derived for processes that cannot reach the boundary. Although the main objective for us is to study the Dupire equation for models with a degenerate behaviour at spatial infinity, we point out that our study also covers the case in which the underlying process may reach zero. Since knowing the distribution of the stock price is equivalent to knowing all call option prices, this provides insight in what boundary conditions to impose on the forward equation for densities, compare [15].

In Section 2 we present the local volatility model, and we state our main result Theorem 2.2 about existence and uniqueness of solutions to the Dupire equation. A discussion how to use Theorem 2.2 for calibration of models is given in Section 3. Finally, Section 4 contains the proof of Theorem 2.2.

\section{Dupire's EQUation for Bubbles}

We let the risk free rate be a constant $r \geq 0$ and we assume that the stock pays a continuous dividend yield $q \geq 0$. Under the risk neutral measure, the stock price process $X$ is modeled by

$$
\left\{\begin{array}{l}
d X(t)=(r-q) X(t) d t+\sigma(X(t), t) d B(t) \\
X(0)=x
\end{array}\right.
$$

where $\sigma$ is a given local volatility function and $B$ is a standard Brownian motion. The current stock price $x>0$ will throughout the paper be considered a fixed constant. We assume that zero is an absorbing barrier for the process $X$. By Ito's formula, the process $e^{-(r-q) t} X(t)$ is a local martingale, but not necessarily a martingale. Processes $X$ for which $e^{-(r-q) t} X(t)$ is a strict local martingale have been suggested to model financial bubbles, compare [4] and [9].

Hypothesis 2.1. The volatility function $\sigma:(0, \infty) \times[0, \infty) \rightarrow(0, \infty)$ is continuous. Moreover, it is locally Hölder(1/2) in the first variable.

Remark Note that we do not require the volatility to be of at most linear growth at spatial infinity. Thus we allow for example models in which $\sigma(x, t)$ grows at least like $x^{1+\eta}$ for large $x$, where $\eta>0$. In fact, in any such model, the process $e^{-(r-q) t} X(t)$ is a strict local martingale, see [2] and [7]. Also note that, regardless of the growth rate of $\sigma$ at infinity, equation (2) has a unique solution that exists for all $t \geq 0$. Indeed, the linear bound at infinity is usually used to avoid exploding solutions; however, in the present context $X$ is automatically a supermartingale and hence does not explode.

We study the discounted expected values

$$
C(K, T):=e^{-r T} E(X(T)-K)^{+}
$$


and

$$
P(K, T):=e^{-r T} E(K-X(T))^{+}
$$

for different non-negative values of the strike price $K$ and times to maturity $T$. We will refer to these expected values as the prices of call options and put options, respectively.

As mentioned in the introduction, there are some subtleties for the Dupire equation in the presence of bubbles. For example, the call price does not satisfy the classical version (1) of the equation. Indeed, assume for simplicity that $r=q=0$. It follows from equation (3) that the call price $C$ is convex in $K$, so $C_{K K} \geq 0$. On the other hand, it is well-known that $C$ can be smaller than $(x-K)^{+}$for some values of $K$ and $T$, compare for example [7], so there exist points where $C_{T}<0$. Therefore, equation (1) clearly fails. Another issue is the failure of uniqueness of solutions for the Dupire equation. A discussion of this is provided in one of the remarks after Theorem 2.2 below.

Our main result shows that the Dupire equation remains valid for put options, but the uniqueness of solutions is lost in general. We also show how to single out the put option price among all possible solutions. For call options, extra terms involving

$$
m(T)=e^{-r T} E X(T)
$$

appear in the equation, and the option price $C$ is the unique bounded solution. Note that if $e^{-(r-q) t} X(t)$ is a martingale, then $m(T)=x e^{-q T}$ and $m_{T}(T)=-q m(T)$. Consequently, the partial differential equation in (5) reduces to the usual Dupire equation (1). However, if $e^{-(r-q) t} X(t)$ is merely a local martingale, then it is a supermartingale being bounded from below, and $e^{q T} m(T)$ decreases with $T$. In that case, $q m(T)+m_{T}(T)$ gives a negative contribution in (5).

Theorem 2.2. Assume that Hypothesis 2.1 holds. Then the call price $C(K, T)$ is the unique bounded classical solution of the equation

$$
\left\{\begin{array}{l}
C_{T}=\mathcal{L} C+q m+m_{T} \quad \text { for }(K, T) \in(0, \infty)^{2} \\
C(K, 0)=(x-K)^{+} \\
C(0, T)=m(T) .
\end{array}\right.
$$

The put price $P(K, T)$ is a classical solution of

$$
\left\{\begin{array}{l}
P_{T}=\mathcal{L} P \quad \text { for }(K, T) \in(0, \infty)^{2} \\
P(K, 0)=(K-x)^{+} \\
P(0, T)=0
\end{array}\right.
$$

Moreover, $P$ is the unique classical solution of (6) satisfying

$$
\left(e^{-r T} K-e^{-q T} x\right)^{+} \leq P(K, T) \leq e^{-r T} K
$$

for all $(K, T) \in(0, \infty)^{2}$.

Remark Equation (6) can formally be viewed as a pricing equation for a call option if we regard $K$ as the spot price of an underlying asset. Consequently, one solution of (6) is given by the stochastic representation

$$
\tilde{P}(K, T)=e^{-q T} E(k(T)-x)^{+},
$$


where $k$ is a diffusion process satisfying

$$
\left\{\begin{array}{l}
d k(t)=-(r-q) k(t) d t+\sigma(k(t), T-t) d B(t) \\
k(0)=K
\end{array}\right.
$$

In the case of bubbles, $\tilde{P}$ is typically not convex in the spatial variable, compare [4] and [7]. However, it follows directly from (3) and (4) that the functions $C$ and $P$ are convex in the strike price $K$. Thus we note that $\tilde{P}$ does not coincide with $P$ for models with bubbles, so there is no uniqueness of solutions to equation (6) in the class of functions of at most linear growth. (If appropriate bounds on the volatility are imposed at zero and at infinity, then $P$ and $\tilde{P}$ coincide, see [13].)

\section{CAlibration OF THE LOCAL VOLATILity FROM OPtion PRICES}

In this section we show how to calibrate a local volatility model from given option data using Theorem 2.2. The calibration in terms of a local volatility model can be done even if the true model is not of the type (2). Indeed, assume for simplicity that $r=q=0$ and that the market uses a pricing measure under which the stock price is a local martingale $\tilde{X}(t)$ with respect to a filtration $\left\{\mathcal{F}_{t}, t \geq 0\right\}$. Moreover, $\tilde{X}$ is absorbed at zero, it has mean $\tilde{m}(T)$ and $\tilde{X}(0)=x$. We emphasize that $\tilde{X}$ may be non-Markovian and may also have jumps. The corresponding call prices are

$$
\tilde{C}(K, T)=E(\tilde{X}(T)-K)^{+} .
$$

We assume that $\tilde{C}(K, T)$ is known for all $(K, T) \in[0, \infty)^{2}$, or more realistically, that a surface has been constructed from a discrete set of available observations. Note that $\tilde{m}(T)=\tilde{C}(0, T)$, so the knowledge of $\tilde{C}$ implies the knowledge of $\tilde{m}$. Inspired by (5), define

$$
\sigma(K, T)=\sqrt{\frac{2\left(\tilde{C}_{T}(K, T)-\tilde{m}_{T}(T)\right)}{\tilde{C}_{K K}(K, T)}} .
$$

Note that if $\tilde{X}_{T}$ has a positive density, then the density equals $\tilde{C}_{K K}$, and the denominator in (8) is thus positive. Similarly, let $T_{1}<T_{2}$ and $g(y):=$ $(y-K)^{+}-y$. Then

$$
\begin{aligned}
E g\left(\tilde{X}\left(T_{2}\right)\right) & =E\left[E\left[g\left(\tilde{X}\left(T_{2}\right)\right) \mid \mathcal{F}_{T_{1}}\right]\right] \geq E\left[g\left(E\left[\tilde{X}\left(T_{2}\right) \mid \mathcal{F}_{T_{1}}\right]\right)\right] \\
& \geq E g\left(\tilde{X}\left(T_{1}\right)\right) .
\end{aligned}
$$

Here we used Jensen's inequality, the fact that $\tilde{X}$ is a supermartingale and that $g$ is convex and decreasing. Therefore, $\tilde{C}_{T}(K, T)-\tilde{m}_{T}(T)$ is positive (if it exists), and the expression for $\sigma$ in (8) is well-defined in the sense of measures.

Theorem 3.1. Assume that $\sigma$ in (8) satisfies Hypothesis 2.1, and let $r=$ $q=0$. For this $\sigma$, define $X$ by (2), and let the corresponding call prices $C(K, T)$ be defined by (3). Then $C \equiv \tilde{C}$.

Proof. First note that both $C$ and $\tilde{C}$ satisfy (5), but with possibly different terms $m$ and $\tilde{m}$. By uniqueness of solutions to (5), it is sufficient to show that $\tilde{m}(T)=m(T):=E X(T)$ for all $T \geq 0$. 
Using the put-call parity, let

$$
\tilde{P}(K, T)=E(K-\tilde{X}(T))^{+}=\tilde{C}(K, T)+K-\tilde{m}(T),
$$

and note that

$$
\sigma(K, T)=\sqrt{\frac{2\left(\tilde{C}_{T}(K, T)-\tilde{m}_{T}(T)\right)}{\tilde{C}_{K K}(K, T)}}=\sqrt{\frac{2 \tilde{P}_{T}(K, T)}{\tilde{P}_{K K}(K, T)}} .
$$

Thus $\tilde{P}$ is a classical solution of (6) also satisfying (7), so by uniqueness of solutions we find that $P=\tilde{P}$, where $P$ is defined by (4). This shows that the distributions of $X(T)$ and $\tilde{X}(T)$ coincide, so in particular $m(T)=\tilde{m}(T)$. As explained in the beginning of the proof, the theorem follows.

Let $\tilde{X}$ be a process satisfying $d \tilde{X}(t)=\beta(t) d t+\gamma(t) d W(t)$ for some bounded and adapted processes $\beta$ and $\gamma$. In [8] it is shown that if $\gamma$ is bounded uniformly away from zero, then one can find a diffusion process $X$ with the same distribution at each time $t \geq 0$. The same problem is studied for jump-diffusion processes in [1]. In both these papers, the diffusion coefficient is bounded. In the case of processes with no drift term, Theorem 3.1 generalizes the results in [8] and [1] to general local martingales (provided that $\sigma$ defined in (8) satisfies Hypothesis 2.1).

\section{Proof of Theorem 2.2}

We prove Theorem 2.2 in several steps.

Step 1. First assume that $\sigma$ satisfies the bounds

$$
D^{-1} x \leq|\sigma(x, t)| \leq D x
$$

for some constant $D>0$ and has bounded derivatives of all orders. By Ito's formula, the process $Y(t):=\ln X(t)$ satisfies

$$
d Y(t)=\beta_{Y}(Y(t), t) d t+\sigma_{Y}(Y(t), t) d B(t),
$$

where

and

$$
\beta_{Y}(y, t):=-\frac{\sigma^{2}\left(e^{y}, t\right)}{2 e^{2 y}}+r-q
$$

$$
\sigma_{Y}(y, t):=\frac{\sigma\left(e^{y}, t\right)}{e^{y}} .
$$

The process $Y$ is a diffusion on the real line with the drift and the volatility possessing bounded derivatives of all orders, and the volatility is bounded from below. Consequently, $Y$ has a smooth transition density

$$
p_{Y}(z, T):=P(Y(T) \in d z) / d z
$$

which satisfies the forward equation

$$
\left(p_{Y}\right)_{T}=\left(\frac{\sigma_{Y}^{2}}{2} p_{Y}\right)_{z z}-\left(\beta_{Y} p_{Y}\right)_{z}
$$

compare for example [16], and $p_{Y}(y, T)$ and its derivatives decay like $o\left(e^{-|y|}\right)$ for large $|y|$. It follows that also the process $X$ has a smooth density $p(y, T)=P(X(T) \in d y) / d y$ which satisfies

$$
p_{T}=\left(\frac{\sigma^{2}}{2} p\right)_{y y}-((r-q) y p)_{y}
$$


Now, since

$$
\begin{aligned}
P(K, T) & =e^{-r T} E(K-X(T))^{+}=e^{-r T} \int_{0}^{K}(K-y) p(y, T) d y \\
& =e^{-r T} \int_{0}^{K} \int_{0}^{y} p(z, T) d z d y
\end{aligned}
$$

by integration by parts, the put price $P(K, T)$ is smooth on $(0, \infty)^{2}$. Straightforward differentiation shows that

$$
\begin{aligned}
P_{T}(K, T)= & e^{-r T} \int_{0}^{K} \int_{0}^{y} p_{T}(z, T) d z d y-r P(K, T) \\
= & e^{-r T} \int_{0}^{K} \int_{0}^{y}\left(\frac{\sigma^{2}(z, T)}{2} p(z, T)\right)_{z z}-(r-q)(z p(z, T))_{z} d z d y \\
& -r P(K, T) \\
= & \frac{\sigma^{2}(K, T)}{2} e^{-r T} p(K, T)-\int_{0}^{K}(r-q) y p(y, T) d y-r P(K, T) \\
= & \frac{\sigma^{2}(K, T)}{2} e^{-r T} p(K, T)-(r-q) K e^{-r T} \int_{0}^{K} p(y, T) d y \\
& +(r-q) e^{-r T} \int_{0}^{K} \int_{0}^{y} p(z, T) d z-r P(K, T) \\
= & \frac{\sigma^{2}(K, T)}{2} e^{-r T} p(K, T)-(r-q) K e^{-r T} \int_{0}^{K} p(y, T) d y \\
& -q P(K, T) .
\end{aligned}
$$

Since $P_{K}(K, T)=e^{-r T} \int_{0}^{K} p(y, T) d y$ and $P_{K K}(K, T)=e^{-r T} p(K, T)$, we find that

$$
P_{T}(K, T)=\frac{\sigma^{2}(K, T)}{2} P_{K K}(K, T)-(r-q) K P_{K}(K, T)-q P(K, T) .
$$

Step 2. Next we carry out an approximation argument to remove the bound (9) for small values of the underlying. Thus we assume that $\sigma$, in addition to Hypothesis 2.1, satisfies

$$
0<\sigma(x, t) \leq D(1+x)
$$

for all $(x, t) \in(0, \infty) \times[0, \infty)$, and we assume that zero is an absorbing boundary for the corresponding solution $X$ of (2). Let $\left\{\sigma_{n}\right\}_{n=1}^{\infty}$ be a sequence of volatilities such that

- $\sigma_{n}(x, t) \rightarrow \sigma(x, t)$ as $n \rightarrow \infty$ for all $(x, t)$,

- each $\sigma_{n}$ satisfies the bound (9) for some constant $D_{n}>0$ and has bounded derivatives of all orders,

- $\sigma_{n}$ satisfies the bound (11) uniformly in $n$, and has a Hölder norm (in the spatial variable) which is bounded on compact subsets of $(0, \infty)^{2}$ uniformly in $n$.

Let $X^{n}$ be the solution of (2) with $\sigma$ replaced by $\sigma_{n}$, and let $P^{n}$ be defined by

$$
P^{n}(K, T)=e^{-r T} E\left(K-X^{n}(T)\right)^{+} .
$$


By Theorem 6 in [10], it follows that $P^{n}(K, T) \rightarrow P(K, T)$ for each $(K, T) \in$ $[0, \infty) \times[0, T]$. By Step 1 above, each $P_{n}$ satisfies

$$
P_{T}^{n}(K, T)=\frac{\sigma_{n}^{2}(K, T)}{2} P_{K K}^{n}(K, T)-(r-q) K P_{K}^{n}(K, T)-q P^{n}(K, T)
$$

on $(0, \infty)^{2}$. Interior Schauder estimates, see [3] or [14], then show that $P$ solves (10).

Step 3. Now we consider the general case of a volatility $\sigma$ that merely satisfies the requirements in Hypothesis 2.1. Let $\left\{\sigma_{n}\right\}_{n=1}^{\infty}$ be a sequence of volatilities satisfying Hypothesis 2.1 and the growth assumption (11) for constants $D_{n}$. Moreover, we assume that $\sigma_{n}(x, t)=\sigma(x, t)$ for $x \leq n$. Let $X^{n}$ be the corresponding stock price process. Since $\sigma_{n}$ coincides with $\sigma$ on $(0, n) \times[0, \infty)$, the random variables $X^{n}(T)$ converge almost surely to $X(T)$. Thus $P^{n}(K, T)$ converges to $P(K, T)$ by bounded convergence. Another application of the interior Schauder estimates shows that $P$ solves (10).

Step 4. Since $e^{-(r-q) t} X(t)$ is a supermartingale, it follows from Jensen's inequality that

$$
\begin{aligned}
P(K, T) & =e^{-r T} E(K-X(T))^{+} \geq e^{-r T}(K-E X(T))^{+} \\
& \geq\left(e^{-r T} K-x e^{-q T}\right)^{+} .
\end{aligned}
$$

On the other hand, we clearly have $P(K, T) \leq e^{-r T} K$. It follows that $P$ is continuous up to the boundary $K=0$ and that $P(0, T)=0$. Moreover, since the paths of $X$ are continuous, we have that $X(T) \rightarrow x$ as $T \downarrow 0$. Therefore, another application of bounded convergence shows that $P(K, T)$ is continuous up to the initial boundary $T=0$, and $P(K, 0)=(K-x)^{+}$. This finishes the proof that the put option price $P$ is a classical solution of (6) that satisfies (7).

Step 5. Next we apply maximum principle techniques to prove that $P$ is the unique classical solution of (6) that satisfies (7). To do that, assume that $P^{1}$ and $P^{2}$ both satisfy (6) and (7). Then $P(K, T):=P^{1}(K, T)-P^{2}(K, T)$ is a bounded classical solution of

$$
\left\{\begin{array}{l}
P_{T}(K, T)=\mathcal{L} P(K, T) \quad \text { for }(K, T) \in(0, \infty)^{2} \\
P(0, T)=0 \\
P(K, 0)=0
\end{array}\right.
$$

Define

$$
h(K)=1+K,
$$

and note that

$$
h_{T}-\mathcal{L} h=r K+q>0 .
$$

For $\epsilon>0$, define

$$
P^{\epsilon}(K, T)=P(K, T)+\epsilon h(K),
$$

let $\Gamma:=\left\{(K, T) \in[0, \infty) \times[0, \bar{T}]: P^{\epsilon}<0\right\}$ for some $\bar{T}>0$, and assume that $\Gamma \neq \emptyset$. Since $P$ is bounded and $P(0, T)=0$, the set $\Gamma$ is contained 
in $\left(D^{-1}, D\right) \times[0, \bar{T}]$ for some constant $D>0$. Thus, by compactness, the infimum

$$
T_{0}:=\inf \{T \geq 0:(K, T) \in \bar{\Gamma} \text { for some } K \in(0, \infty)\}
$$

is attained at some point $\left(K_{0}, T_{0}\right)$. Since $P^{\epsilon}(K, 0)=\epsilon h(K)>0$, we have $T_{0}>0$. Therefore, at the point $\left(K_{0}, T_{0}\right)$ we have

$$
P_{T}^{\epsilon}\left(K_{0}, T_{0}\right)-\mathcal{L} P^{\epsilon}\left(K_{0}, T_{0}\right)=\epsilon\left(h_{T}-\mathcal{L} h\right)\left(K_{0}, T_{0}\right)>0 .
$$

On the other hand, by the definition of $T_{0}$, the function $P^{\epsilon}$ satisfies $P^{\epsilon}=0$, $P_{K}^{\epsilon}=0, P_{K K}^{\epsilon} \geq 0$ and $P_{T}^{\epsilon} \leq 0$ at the point $\left(K_{0}, T_{0}\right)$. Consequently,

$$
P_{T}^{\epsilon}\left(K_{0}, T_{0}\right)-\mathcal{L} P^{\epsilon}\left(K_{0}, T_{0}\right) \leq 0 .
$$

This contradiction shows that $\Gamma=\emptyset$, so $P^{\epsilon} \geq 0$ on $(0, \infty) \times[0, \bar{T}]$. Since $\epsilon>0$ and $\bar{T}$ are arbitrary, it follows that $P \geq 0$. Interchanging the role of $P_{1}$ and $P_{2}$ yields the reverse inequality, i.e. $P_{1}=P_{2}$.

Step 6. Finally, we treat the call option price $C$ using a put-call parity relation. Taking expected values in the equality

$$
(X(T)-K)^{+}=(K-X(T))^{+}-K+X(T)
$$

we find that

$$
C(K, T)=P(K, T)-e^{-r T} K+m(T) .
$$

Therefore,

$$
\begin{aligned}
C_{T}(K, T)= & P_{T}(K, T)+r e^{-r T} K+m_{T}(T) \\
= & \frac{\sigma^{2}(K, T)}{2} P_{K K}(K, T)-(r-q) K P_{K}(K, T)-q P(K, T) \\
& +r e^{-r T} K+m_{T}(T) \\
= & \frac{\sigma^{2}(K, T)}{2} C_{K K}(K, T)-(r-q) K C_{K}(K, T)-q C(K, T) \\
& +q m(T)+m_{T}(T),
\end{aligned}
$$

where we used (12), $P_{K}=C_{K}+e^{-r T}$ and $P_{K K}=C_{K K}$. The fact that $C$ satisfies the given boundary conditions also follows from the put-call parity (12) and the boundary behaviour of $P$. Finally, the proof of the uniqueness of the solutions to equation (6) within the given class also shows uniqueness of solutions with a bounded difference to $e^{-r T} K$. This translates directly to uniqueness for equation (5) for bounded functions. This finishes the proof of Theorem 2.2.

\section{REFERENCES}

[1] Bentata, A. and Cont, R. Matching marginal distributions of a semimartingale with a Markov process. C. R. Acad. Sci. Paris, Ser. I 347 (2009).

[2] Blei, S. and Engelbert, H.-J. On exponential local martingales associated with strong Markov continuous local martingales, Stoch. Proc. Appl. 119 (2009), 28592880.

[3] Brandt, A. Interior Schauder estimates for parabolic differential- (or difference-) equations via the maximum principle. Israel J. Math. 7 (1969), 254-262.

[4] Cox, A. and Hobson, D. Local martingales, bubbles and option prices, Finance Stoch. 9 (2005), 477-492.

[5] Dupire B., Pricing with a smile, Risk (1994) 18-20. 
[6] Ekström, E., Lötstedt, P., Tysk, J. and von Sydow, L. Numerical option pricing in the presence of bubbles. Preprint (2008).

[7] Ekström, E. and Tysk, J. Bubbles, convexity and the Black-Scholes equation. Ann. Appl. Probab. 19 (2009), 1369-1384.

[8] Gyöngy, I. Mimicking the one-dimensional marginal distributions of processes having an Itô differential. Probab. Theory Relat. Fields 71 (1986), 501-516.

[9] Heston, S., Loewenstein, M., Willard, G. Options and bubbles, Rev. Financ. Stud. 20 (2007), 359-390.

[10] Janson, S., and Tysk, J. Volatility time and properties of option prices, Ann. Appl. Probab. 13 (3) (2003) 890-913.

[11] Jarrow, R. A., Protter, P. and Shimbo, K. Asset price bubbles in complete markets. In Advances in Mathematical Finance 105-130. Birkhäuser, Boston 2006.

[12] Jarrow, R. A., Protter, P. and Shimbo, K. Asset price bubbles in incomplete markets. Preprint (2007).

[13] Jourdain, B. Stochastic flow approach to Dupire's formula. Finance Stoch. 11 (2007), no. 4, 521-535.

[14] Knerr, B. F. Parabolic interior Schauder estimates by the maximum principle, Arch. Rational Mech. Anal. 75 (1980/81), 51-58.

[15] Lucic, V. Boundary Conditions for Computing Densities in Hybrid Models via PDE Methods. Preprint (2008).

[16] Rogers, L.C.G., Smooth transition densities for one-dimensional diffusions, Bull. London Math. Soc. 17 (1985), 157-161.

Department of Mathematics, Uppsala University, Box 480, SE-751 06 UppSALA, SWEDEN

E-mail address: ekstrom@math.uu.se, johant@math.uu.se 